\title{
Marcadores de estrés oxidativo en el diagnóstico de artritis reumatoide
}

\section{Oxidative stress markers in the diagnosis of rheumatoid arthritis}

\author{
M. Laíño Piñeiro, E. Ornilla Laraudogoitia
}

\section{Sr. Director:}

Hemos leído con interés el trabajo publicado en su revista por Melguizo y col referente a la "Utilidad diagnóstica de los marcadores de estrés oxidativo en artritis reumatoide precoz en pacientes no fumadores y anti-CCP negativos" ${ }^{\text {. Nos gusta- }}$ ría hacer algún comentario al mismo. En primer lugar, estamos de acuerdo en la importancia de incorporar nuevos marcadores para el diagnóstico precoz de la artritis reumatoide, porque un diagnóstico temprano de la enfermedad permitirá un mejor pronóstico a corto y largo plazo al poder instaurar el tratamiento en fases iniciales de la enfermedad ${ }^{2}$. Nos parece muy acertado realizar los subgrupos teniendo en cuenta el hábito tabáquico de los sujetos, porque el tabaco es una causa de estrés oxidativo y es un factor de riesgo independiente de artritis reumatoide ${ }^{3}$. Sin embargo, el estrés oxidativo también es importante en otras enfermedades, como la aterosclerosis, diabetes mellitus e insuficiencia renal ${ }^{4}$. Asimismo, también es responsable de situaciones fisiológicas como el envejecimiento. En este sentido, en el estudio no se especifica si los pacientes tienen comorbilidades asociadas, que también podrían incrementar los marcadores de estrés oxidativo.
Es importante tener en cuenta las causas de variabilidad en la determinación de dichos marcadores e intentar minimizarlos antes de utilizarlos en práctica clínica habitual. Es conocida la influencia de la dieta en los marcadores de estrés oxidativo ${ }^{5}$. El consumo de aceites y grasa sometidos a sucesivos calentamientos influye sobre la peroxidación lipídica plasmática y es mayor cuanto mayor sea el número de calentamientos realizados. Hoy en día, los hábitos dietéticos incluyen una proporción elevada de productos fritos, procesados o la llamada comida rápida, incrementado el estrés oxidativo. Por lo tanto, hay que tomar con cautela los resultados aportados y se requerirán más estudios antes de poder utilizarlos en el diagnóstico habitual de la artritis reumatoide.

\section{BIBLIOGRAFÍA}

1. Melguizo E, Navarro V, Hernández B, Santos K, Arrobas T, Domínguez C, et al. Diagnostic utility of oxidative damage markers for early rheumatoid artritis in non-somkers and negative anti-CCP patients. An Sist Sanit Navar 2014; 1: 109-115.

2. Van Nies JA, Krabben A, Schoones JW, Huizinga TW, Kloppenburg M, van der Helm-VAn Mil AH. What is the evidence for the presence of a
Unidad de Reumatología. Clínica Universidad de Navarra. Pamplona.
Recepción: 24 de noviembre de 2014

Aceptación definitiva: 24 de noviembre de 2014 
therapeutic window of opportunity in rheumatoid arthritis? A systematic literature review. Ann Rheum Dis 2014; 73: 5 861-870.

3. WolfE F. The effect of smoking on clinical, laboratory, and radiographic status in rheumatoid arthritis. J Rheumatol 2000; 27: 630637.
4. EleJALDE GuerRa JI. Estrés oxidativo, enfermedades y tratamientos antioxidantes. An Med Interna 2001; 18, 326-335.

5. Barbosa KBF., Bressan J, Zulet MA., Martínez JA. Influencia de la dieta sobre marcadores plasmáticos de estrés oxidativo en humanos. An Sist Sanit Navar 2008; 31: 259-280. 